\title{
First principles calculations of structural, electronic and thermal properties of lead chalcogenides PbS, PbSe and PbTe compounds
}

\author{
N BOUKHRIS ${ }^{1}$, H MERADJI ${ }^{1, *}$, S AMARA KORBA ${ }^{1}$, S DRABLIA ${ }^{1}$, S GHEMID ${ }^{1}$ and \\ F EL HAJ HASSAN ${ }^{2}$ \\ ${ }^{1}$ Laboratoire LPR, Département de Physique, Faculté des Sciences, Université Badji Mokhtar, Annaba 23000, Algeria \\ ${ }^{2}$ Laboratoire de Physique des Matériaux, Faculté des Sciences, Université Libanaise, Elhadath, Beirut 6573-14, Lebanon
}

MS received 28 July 2013; revised 14 October 2013

\begin{abstract}
The structural, electronic and thermal properties of lead chalcogenides PbS, PbSe and BeTe using full-potential linear augmented plane wave (FP-LAPW) method are investigated. The exchange-correlation energy within the local density approximation (LDA) and the generalized gradient approximation (GGA) are described. The calculated structural parameters are in reasonable agreement with the available experimental and theoretical data. The electronic band structure shows that the fundamental energy gap is direct $(\mathrm{L}-\mathrm{L})$ for all the compounds. Thermal effects on some macroscopic properties of these compounds are predicted using the quasi-harmonic Debye model, in which the lattice vibrations are taken into account. The variations of the lattice constant, bulk modulus, heat capacity, volume expansion coefficient and Debye temperature with temperature and pressure are obtained successfully. The effect of spin-orbit interaction is found to be negligible in determining the thermal properties and leads to a richer electronic structure.
\end{abstract}

Keywords. Lead chalcogenides; band-structure; Debye model.

\section{Introduction}

During the past decades, the lead chalcogenides, $\mathrm{PbX}$ $(X=S$, Se and $T e$ ) have been a subject of a great amount of theoretical and experimental studies, motivated by their importance in infrared technology, and more recently, because of their utility in laser technology and as thermoelectric materials. The PbX compounds are narrow direct gap semiconductors (group IV-VI), which crystallize at ambient conditions in the cubic $\mathrm{NaCl}$ structure. The lead salts exhibit properties which are unusual, relative to other semiconductors. Compared for example, with the usual III-V compounds, these IV-VI chalcogens present non-typical electronic and transport properties, such as higher carrier mobilities, higher dielectric constants, narrow band gaps and positive temperature coefficients (Cowley 1965; Dalven 1973; Murase 1980). These properties make them potential candidates for different technological applications. They have been used in thermoelectronic, optoelectronic or spintronic devices, especially in long wavelength imaging (Hummer et al 2007), infrared diode lasers (Preier 1979) and in thermophotovoltaic energy converters (Zogg et al 1994). The semiconductors $\mathrm{PbS}, \mathrm{PbSe}$ and $\mathrm{PbTe}$ show a small direct gap at the L point of the Brillouin zone. In contrast to most of the semiconductors, this gap decreases with hydrostatic

\footnotetext{
*Author for correspondence (hmeradji@yahoo.fr)
}

pressure (Andreev 1968; Besson et al 1968; Martinez 1973) and increases with temperature (Tauber et al 1966; Andreev 1968; Martinez 1973). Although, considerable progress has been made concerning theoretical description of the structural and electronic properties of lead chalcogenides compounds, there is a real lack of knowledge of their thermal properties. An accurate description of thermal properties of solids is crucial, since, it plays a significant role in determining various material properties. To the best of our knowledge, there are no theoretical reports on the thermal behaviour of these compounds in the literature. Consequently, the primary purpose of this work is to provide some additional information to the existing data on the physical properties of lead chalcogenides using first-principles calculations. The electronic structure calculations are performed to derive the necessary parameters and the effect of spin-orbit interaction is examined. The paper is organized as follows: after a brief introduction in $\S 1$, the theoretical framework within which all the calculations have been performed is outlined in $\S 2$. In $\S 3$, we present and discuss the results of our study. A conclusion of the present investigation is given in $\S 4$.

\section{Computational method}

The calculations reported in this work were done with the WIEN2K programme developed by Blaha et al (2008). 
This programme uses the full-potential linearized augmented plane wave (FP-LAPW) method (Anderson 1975) based on density functional theory (Hohenberg and Kohn 1964; Kohn and Sham 1965). In this method, the space is divided into an interstitial region (IR) and nonoverlapping (MT) spheres centred at the atomic sites. In order to treat the exchange correlation $(x c)$ potential, we used both the local density approximation (LDA) as parameterized by Perdew and Wang (1986) and the generalized gradient approximation (GGA) as parameterized by Perdew et al (1996). In the FP-LAPW method, the space is divided into an interstitial region (IR) and non-overlapping muffin-tin (MT) spheres centred at the atomic sites. In the IR region, the basis set consists of plane waves. Inside the MT spheres, the basis set is described by radial solutions of one particle Schrödinger equation (at fixed energy) and their energy derivatives multiplied by spherical harmonics. A plane wave cut-off of $k_{\max }=$ $8 / R_{\mathrm{MT}}$ (where $R_{\mathrm{MT}}$ is the smallest muffin-tin radius in the unit cell) was used. The valence wave functions inside the muffin-tin spheres are expanded up to $l_{\max }=10$, while the charge density was Fourier expanded up to $G_{\max }=$ 14 (Ryd $^{1 / 2}$. The muffin-tin radii are taken to be $2 \cdot 2$ a.u. for $\mathrm{Pb}$ atom and 2 a.u. for $\mathrm{S}$, Se and Te atoms. The elastic constants have been calculated using the method recently developed by Charpin and integrated in the WIEN2K package (Blaha et al 2008). A mesh of 47 special $k$-points for PbS, PbSe and PbTe compounds is taken in the irreducible wedge of the Brillouin zone for energy calculation. Both the plane wave cut-off and the number of $k$-points were varied to ensure total energy convergence. The study of thermal effects was done within the quasiharmonic Debye model implemented in the Gibbs program (Blanco et al 2004). The quasi-harmonic Debye model allows us to obtain all thermodynamic quantities from the calculated energy-volume points. The common thermodynamic parameters that depend on temperature and pressure are used to derive other macroscopic properties.

\section{Results and discussion}

\subsection{Structural and elastic properties}

The fitting of the Murnaghan (1944) equation of state to the total energies vs unit cell volumes, yields to the equilibrium lattice parameter $(a)$, bulk modulus $(B)$ and the pressure derivative of the bulk modulus $B^{\prime}$. The obtained results for all the systems are presented in table 1 , along with some previous theoretical calculations and available experimental measurements. As usual, the obtained lattice parameters are larger for the GGA approach than for the LDA approach. On the contrary, the GGA values for the bulk modulus are smaller than the LDA ones. When we analyse these results, we find that there is a good agreement between our results and the reported theoretical and experimental investigations. The spin-orbit interaction does not affect the equilibrium properties, in fact, the GGA values obtained for the lattice parameter introducing this interaction in our calculations are 6.009, 6.222 and 6.577 $\AA$, for PbS, PbSe and PbTe compounds, respectively. Albanesi et al (2000), using FP-LAPW method within GGA approximation and considering spinorbit effect, reported lattice parameters for PbSe and PbTe of 6.23 and $6.57 \AA$, respectively, which are close to our obtained values which confirm the above statement.

Elastic properties of a solid are important because they relate to various fundamental solid-state phenomena such as interatomic bonding, equations of state and phonon spectra. Most importantly, knowledge of elastic constants is essential for many practical applications related to the mechanical properties of a solid: load deflection, thermoelastic stress, internal strain, sound velocities and fracture toughness. Therefore, knowledge of the elastic properties of $\mathrm{PbS}$, PbSe and PbTe will be of great interest in understanding their behaviour under different constraints. A cubic crystal has three different symmetry elements $\left(C_{11}\right.$, $C_{12}$ and $\left.C_{44}\right)$. The calculated elastic constants for the compounds, within LDA and GGA, are given in table 1, which also contains results of previous calculations as well as the experimental data.

For these lead chalcogenides, the present results for $C_{11}$ are in good agreement with the experimental values, when we use the GGA approximation. The $C_{44}$, which reflects the resistance to shear deformation, is lower than $C_{11}$, which is related to the unidirectional compression along the principal crystallographic directions, indicating the weak resistance to shear deformation compared to the resistance to the unidirectional compression. A more extensive comparison can be made for the bulk modulus, $B$, which is often reported in the literature and which we can calculate from our values of $C_{11}$ and $C_{12}$. The bulk modulus calculated from the theoretical values of the elastic constants, $B=\left(C_{11}+2 C_{12}\right) / 3$ is also listed in table 1. The bulk modulus calculated from the total energy minimization and from the elastic constants have nearly the same value. The requirement of mechanical stability in a cubic crystal leads to the following restrictions on the elastic constants: $C_{11}-C_{12}>0, C_{11}>0, C_{44}>0, C_{11}+$ $2 C_{12}>0$. The elastic constants in table 1 obey these stability conditions, including the fact that $C_{12}$ must be smaller than $C_{11}$. Our calculated elastic constants also obey the cubic stability conditions, meaning that $C_{12}<B<C_{11}$.

\subsection{Electronic properties}

We now turn our attention to the electronic properties, such as the direct energy band gaps. For this purpose, the band structures of PbS, PbSe and PbTe compounds were obtained within the GGA. We include the spin-orbit 
Table 1. Lattice constant $a$, bulk modulus $B$, pressure derivative of bulk modulus $B^{\prime}$ and elastic constant parameters of PbS, PbSe and PbTe compared with the experimental data and other theoretical works.

\begin{tabular}{|c|c|c|c|c|c|}
\hline & \multicolumn{2}{|c|}{ Present work } & \multirow[b]{2}{*}{ Experiment } & \multicolumn{2}{|c|}{ Other theoretical works } \\
\hline & LDA & GGA & & LDA & GGA \\
\hline \multicolumn{6}{|l|}{$\mathrm{PbS}$} \\
\hline$a(\AA)$ & $5 \cdot 836$ & $6 \cdot 010$ & $5 \cdot 929^{\mathrm{a}}, 5 \cdot 936^{\mathrm{c}, \mathrm{d}}, 5 \cdot 940^{\mathrm{f}}$ & $5.860^{\mathrm{b}}, 5.906^{\mathrm{e}}, 5.854^{\mathrm{g}}$ & $6 \cdot 012^{b, g}$ \\
\hline$B(\mathrm{GPa})$ & $63 \cdot 926$ & $53 \cdot 384$ & $52 \cdot 9^{\mathrm{a}}$ & $64 \cdot 8^{\mathrm{b}}, 66 \cdot 3^{\mathrm{e}}, 67 \cdot 3^{\mathrm{g}}$ & $53 \cdot 3^{\mathrm{b}}, 53 \cdot 2^{\mathrm{g}}$ \\
\hline$B^{\prime}$ & $4 \cdot 253$ & 4 & & & \\
\hline$C_{11}(\mathrm{GPa})$ & $169 \cdot 3$ & $123 \cdot 4$ & $124^{\mathrm{a}}$ & $172^{\mathrm{b}}$ & $136^{\mathrm{b}}$ \\
\hline$C_{12}(\mathrm{GPa})$ & $16 \cdot 70$ & $17 \cdot 2$ & $33^{\mathrm{a}}$ & $11 \cdot 1^{\mathrm{b}}$ & $11 \cdot 9^{\mathrm{b}}$ \\
\hline$C_{44}(\mathrm{GPa})$ & $21 \cdot 3$ & $13 \cdot 1$ & $23^{\mathrm{a}}$ & $20 \cdot 1^{b}$ & $20 \cdot 1^{b}$ \\
\hline$B=\left(C_{11}+2 C_{12}\right) / 3$ & $67 \cdot 56$ & $52 \cdot 46$ & & & \\
\hline \multicolumn{6}{|l|}{ PbSe } \\
\hline$a(\AA)$ & $6 \cdot 030$ & $6 \cdot 210$ & $6 \cdot 117^{\mathrm{a}}, 6 \cdot 124^{\mathrm{c}, \mathrm{d}}, 6 \cdot 130^{\mathrm{f}}$ & $6 \cdot 053^{\mathrm{b}}, 6 \cdot 098^{\mathrm{e}}, 6 \cdot 046^{\mathrm{g}}$ & $6 \cdot 196^{\mathrm{b}}, 6 \cdot 222^{\mathrm{g}}$ \\
\hline$B(\mathrm{GPa})$ & $56 \cdot 692$ & $49 \cdot 187$ & $54 \cdot 1^{\mathrm{a}}$ & $58 \cdot 3^{\mathrm{b}}, 60 \cdot 8^{\mathrm{e}}, 59 \cdot 3^{\mathrm{g}}$ & $49 \cdot 1^{\mathrm{b}}, 47 \cdot 5^{\mathrm{g}}$ \\
\hline$B^{\prime}$ & $3 \cdot 811$ & $3 \cdot 660$ & & & \\
\hline$C_{11}(\mathrm{GPa})$ & $165 \cdot 9$ & 110 & $123 \cdot 7^{\mathrm{a}}$ & $159 \cdot 5^{\mathrm{b}}$ & $120 \cdot 8^{\mathrm{b}}$ \\
\hline$C_{12}(\mathrm{GPa})$ & $5 \cdot 4$ & $14 \cdot 9$ & $19 \cdot 3^{\mathrm{a}}$ & $7 \cdot 8^{\mathrm{b}}$ & $8 \cdot 9^{\mathrm{b}}$ \\
\hline$C_{44}(\mathrm{GPa})$ & $17 \cdot 4$ & 21 & $15 \cdot 9^{a}$ & $18^{\mathrm{b}}$ & $17 \cdot 2^{\mathrm{b}}$ \\
\hline$B=\left(C_{11}+2 C_{12}\right) / 3$ & $58 \cdot 90$ & $46 \cdot 40$ & & & \\
\hline \multicolumn{6}{|l|}{ PbTe } \\
\hline$a(\AA)$ & $6 \cdot 367$ & $6 \cdot 560$ & $6 \cdot 462^{a, c}, 6 \cdot 460^{f}$ & $6 \cdot 37^{\mathrm{b}}, 6 \cdot 44^{\mathrm{e}}, 6 \cdot 383^{\mathrm{g}}$ & $6 \cdot 565^{\mathrm{b}}, 6 \cdot 57^{\mathrm{g}}$ \\
\hline$B(\mathrm{GPa})$ & $48 \cdot 897$ & $38 \cdot 406$ & $39 \cdot 8^{\mathrm{a}}$ & $51 \cdot 4^{\mathrm{b}}, 51 \cdot 7^{\mathrm{e}}, 50 \cdot 2^{\mathrm{g}}$ & $41 \cdot 4^{\mathrm{b}}, 38 \cdot 7^{\mathrm{g}}$ \\
\hline$B^{\prime}$ & $4 \cdot 717$ & $4 \cdot 462$ & & & \\
\hline$C_{11}(\mathrm{GPa})$ & 122 & 107 & $105 \cdot 3^{\mathrm{a}}$ & $144 \cdot 2^{\mathrm{b}}$ & $115 \cdot 7^{\mathrm{b}}$ \\
\hline$C_{12}(\mathrm{GPa})$ & $10 \cdot 7$ & $3 \cdot 2$ & $7^{\mathrm{a}}$ & $1 \cdot 5^{\mathrm{b}}$ & $4 \cdot 2^{\mathrm{b}}$ \\
\hline$C_{44}(\mathrm{GPa})$ & $17 \cdot 6$ & $17 \cdot 2$ & $13 \cdot 2^{\mathrm{a}}$ & $15 \cdot 7^{\mathrm{b}}$ & $14 \cdot 3^{\mathrm{b}}$ \\
\hline$B=\left(C_{11}+2 C_{12}\right) / 3$ & $47 \cdot 80$ & $37 \cdot 8$ & & & \\
\hline
\end{tabular}

${ }^{\mathrm{a}}$ Madelung et al (1983); ${ }^{\mathrm{b}}$ Lach-Hab et al (2002); ${ }^{\mathrm{C}}$ Dalven (1973); ${ }^{\mathrm{d}}$ Cohen and Chelikowsky (1989); ${ }^{\mathrm{e}}$ Wei and Zunger (1997); ${ }^{\mathrm{f}}$ Delin et al (1998) and ${ }^{\mathrm{g}}$ Zaoui et al (2009).

coupling in our calculations by using the second-variation method programmed in the code WIEN2K. It has a significant influence on the band structures, when heavy elements like lead $(\mathrm{Pb})$ are present. The spin-orbit coupling causes splitting of $p$ - and $f$-bands and as a consequence, it will influence the other electronic properties. The valence band maximum (VBM) and the conduction band minimum (CBM) occur at the $L$ point in the Brillouin zone for these binary compounds. The calculated band gap values with both GGA and GGA-so for lead chalcogenide compounds are listed in table 2 along with the available theoretical and experimental results. The obtained GGA band gap values are slightly overestimated compared to the available theoretical results. This discrepancy could be attributed to the different functional parameters used in the calculations. The values of the calculated band gaps with spin-orbit interaction show a significant improvement over the results based on GGA compared to the experimental data. Our calculated GGA-so band gap values are comparable to those obtained by Albanesi et al (2000) using first principles DFT calculations. The spinorbit interaction reduces the band gap values for the herein studied compounds, and similar results have been found for $\mathrm{HgI}_{2}$ and $\mathrm{SnI}_{2}$ (Ahuja et al 1996; Ravindra et al
1997). In general, GGA calculations tend to underestimate the main energy gap of semiconductors by $40-50 \%$. In fact, the energy gaps are systematically underestimated in $a b$ initio calculations and that is an intrinsic feature of density functional theory, DFT being a groundstate theory not suitable for describing excited-state properties, such as the energy gap. However, it is widely accepted that GGA (LDA) electronic band structures are qualitatively in good agreement with experiments as far as ordering of energy levels and shape of bands.

\subsection{Thermal properties}

To investigate the thermal properties of $\mathrm{PbS}, \mathrm{PbSe}$ and PbTe compounds, we apply the quasi-harmonic Debye model (Blanco et al 2004), in which the non-equilibrium Gibbs function $G^{*}(V ; P, T)$ can be written as

$$
G^{*}(V ; P, T)=E(V)+P V+A_{\mathrm{Vib}}[\theta(V) ; T],
$$

where $E(V)$ is the total energy per unit cell, $P V$ the constant hydrostatic pressure condition, $\theta(V)$ the Debye temperature and $A_{\mathrm{Vib}}$ the vibrational term, which can be 
Table 2. Energy band gaps $(\mathrm{L} \rightarrow \mathrm{L})$ in $(\mathrm{eV})$ of $\mathrm{PbS}$, PbSe and PbTe compounds.

\begin{tabular}{|c|c|c|c|c|}
\hline & \multicolumn{2}{|c|}{ Present work } & \multirow[b]{2}{*}{ Experiment } & \multirow[b]{2}{*}{ Other works } \\
\hline & GGA-so & GGA & & \\
\hline $\mathrm{PbS}$ & $0 \cdot 169$ & 0.496 & $0 \cdot 290^{\mathrm{a}}$ & $0 \cdot 380^{\mathrm{b}}, 0.448^{\mathrm{c}}, 0.074^{\mathrm{d}}$ \\
\hline PbSe & 0.072 & 0.425 & $0 \cdot 17^{\mathrm{a}}$ & $0 \cdot 340^{\mathrm{b}}, 0 \cdot 318^{\mathrm{c}}, 0 \cdot 022^{\mathrm{d}}, 0 \cdot 121^{\mathrm{f}}$ \\
\hline PbTe & $0 \cdot 182$ & $0 \cdot 833$ & $0 \cdot 19^{\mathrm{e}}$ & $0 \cdot 737^{\mathrm{b}}, 0.643^{\mathrm{c}}, 0 \cdot 16^{\mathrm{f}}$ \\
\hline
\end{tabular}

${ }^{\mathrm{a}}$ Gupta and Ravindra (1980); ${ }^{\mathrm{b}}$ Lach-Hab et al (2002); ${ }^{\mathrm{c}}$ Zaoui et al (2009); ${ }^{\mathrm{d}}$ Zhang et al (2009);

e Dalven (1973); ${ }^{\mathrm{f}}$ Albanesi et al (2000).

written using the Debye model of the phonon density of states as (Blanco et al 1996; Flórez et al 2002)

$$
A_{\mathrm{Vib}}(\theta ; T)=n k_{\mathrm{B}} T\left[\frac{9 \theta}{8 T}+3 \ln \left(1-e^{-\theta / T}\right)-D(\theta / T)\right],
$$

where $D(\theta / T)$ represents the Debye integral, $n$ the number of atoms per formula unit.

For an isotropic solid, $\theta$ is expressed as (Blanco et al 1996)

$$
\theta_{\mathrm{D}}=\frac{\hbar}{k_{\mathrm{B}}}\left[6 \pi^{2} V^{1 / 2} n\right]^{1 / 3} f(\sigma) \sqrt{\frac{B_{\mathrm{s}}}{M}},
$$

$M$ being the molecular mass per unit cell and $B_{\mathrm{s}}$ the adiabatic bulk modulus, approximated by the static compressibility (Blanco et al 2004)

$$
B_{\mathrm{s}} \cong B(V)=V\left\{\frac{d^{2} E(V)}{\mathrm{d} V^{2}}\right\},
$$

$f(\sigma)$ is given by (Francisco et al 2001; Flórez et al 2002)

$$
f(\sigma)=\left\{3\left[2\left(\frac{21+\sigma}{31-2 \sigma}\right)^{3 / 2}+\left(\frac{11+\sigma}{31-\sigma}\right)^{3 / 2}\right]^{-1}\right\}^{1 / 3} .
$$

The Poisson ratio $\sigma$ is taken as $0 \cdot 25$ (Poirier 2000). Therefore, the non-equilibrium Gibbs function $G^{*}(V ; P$, $T)$ as a function of $(V ; P, T)$ can be minimized with respect to volume $V$

$$
\left[\frac{\partial G^{*}(V: P, T)}{\partial V}\right]_{\mathrm{P}, \mathrm{T}}=0 .
$$

By solving (10), one can get the thermal equation of state (EOS), $V(P, T)$. The isothermal bulk modulus, $B_{\mathrm{T}}$, the heat capacity, $C_{\mathrm{V}}$ and the thermal expansion coefficient, $\alpha$ are given by (Blanco et al 1996)

$$
\begin{aligned}
& B_{\mathrm{T}}(P, V)=V\left(\frac{\delta^{2} G^{*}(V ; P, T)}{\delta V^{2}}\right)_{\mathrm{P}, \mathrm{T}}, \\
& C_{\mathrm{V}}=3 n k_{\mathrm{B}}\left[4 D(\theta / T)-\frac{3 \theta / T}{e^{\theta / T}-1}\right],
\end{aligned}
$$

$$
\alpha=\frac{\gamma C_{\mathrm{V}}}{B_{\mathrm{T}} V},
$$

where $\gamma$ is the Gruneisen parameter, which is defined as

$$
\gamma=-\frac{d \ln \theta(V)}{d \ln V} .
$$

Through the quasi-harmonic Debye model, one could calculate the thermodynamic quantities of any temperature and pressure of compounds from the calculated $E-V$ data at $T=0$ and $P=0$. By using the method above, we can successfully obtain the thermal properties of $\mathrm{PbS}$, $\mathrm{PbSe}$ and $\mathrm{PbTe}$ compounds. The thermal properties are determined in the temperature range from 0 to $1000 \mathrm{~K}$, where the quasi-harmonic model remains fully valid. The pressure effect is studied in the 0-40 GPa range.

The effect of the spin-orbit interaction is found to be negligible in determining the thermal properties since, the calculations of these properties use $E-V$ data which are not influenced by spin-orbit coupling. The temperature effects at several pressures on the lattice parameters and bulk modulus of the compounds are shown in figure 1 . The lattice parameters increase with increasing temperature for a given pressure, but the rate of increase is very moderate, especially at high pressures. At a given temperature, the lattice parameters decrease with increasing pressure. The effect of increasing temperature and decreasing pressure on the lattice parameter are identical. The bulk modulus is related to interatomic potentials because it can be obtained by the second derivatives of the internal energy with respect to strains. The temperature dependence of bulk modulus partially reflects the anharmonic interactions because for a pure harmonic crystal, the bulk modulus would be independent of temperature. At $0 \mathrm{~K}(P=0)$, our calculated bulk modulus for PbS, PbSe and PbTe are 47.69, 46.76 and 34.04 GPa, respectively, which are in good agreement with the values obtained by the WIEN2K code. From figure 1, we can see that when $T<100 \mathrm{~K}, B$ is nearly a constant, which indicates that the lattice parameter nearly keeps constant. When $T>100 \mathrm{~K}$, the bulk modulus decreases as $T$ increases. This indicates that the unit cell volume varies significantly as the temperature increases. The bulk modulus increases with pressure at a given temperature, 

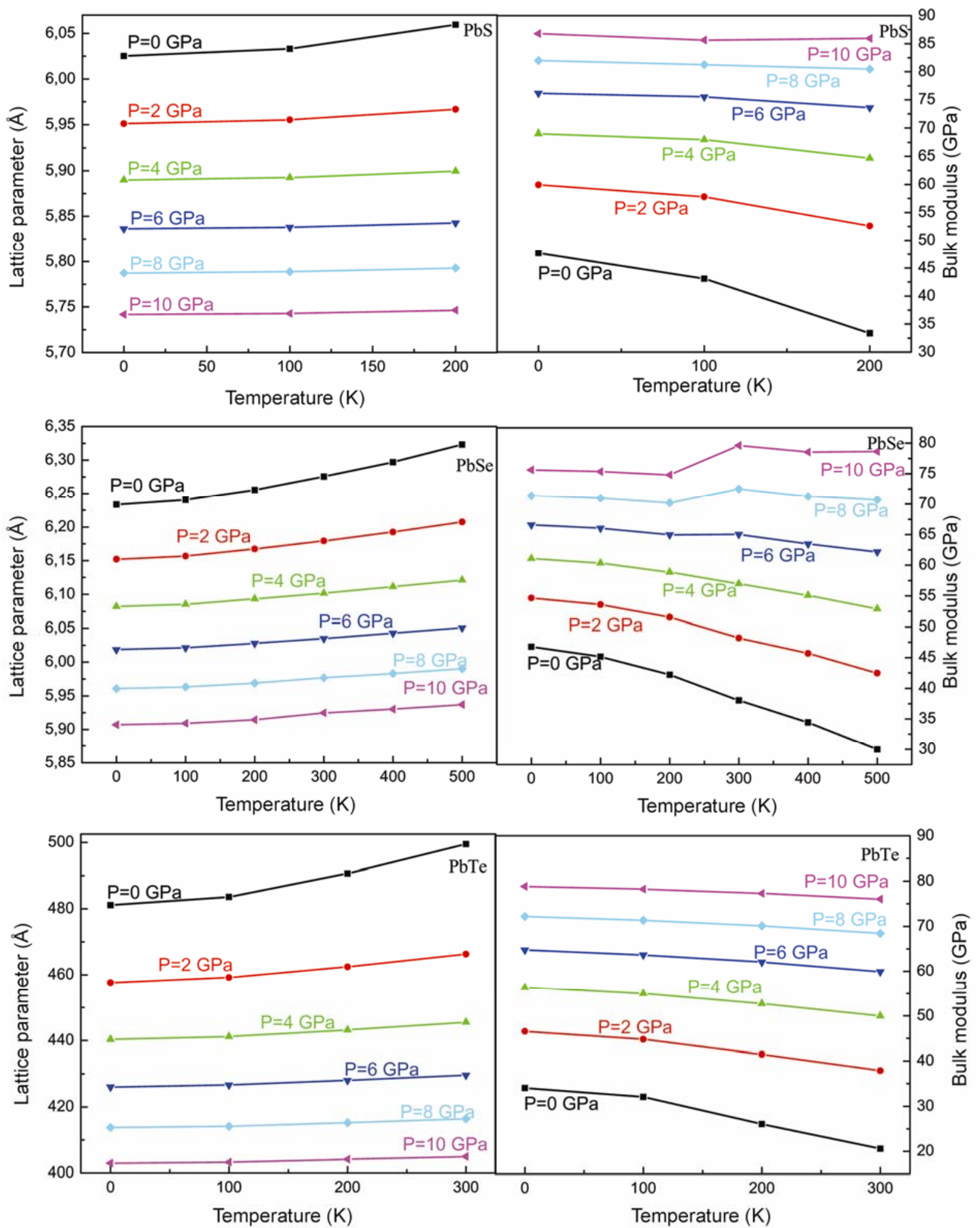

Figure 1. Variation of lattice constant and bulk modulus of PbS, PbSe and $\mathrm{PbTe}$ as a function of temperature at several pressures.

which indicates that the hardness of these compounds decreases with the increasing temperature and increases with applied pressure.

The variation of the heat capacities $C_{\mathrm{v}}$ and $C_{\mathrm{p}}$ with temperature in the case of $\mathrm{PbSe}$ compound are shown in figures 2 and 3. Two famous limiting cases are correctly predicted by the standard elastic continuum theory (Debye 1912). At high temperature, the constant volume heat capacity, $C_{\mathrm{v}}$ tends to the Dulong-Petit limit $50 \mathrm{~J} \mathrm{~mol}^{-1} \mathrm{~K}^{-1}$, whereas $C_{\mathrm{p}}$ follows a linear increase. At 
sufficiently low temperature, $C_{\mathrm{v}}$ and $C_{\mathrm{p}}$ are proportional to $T^{3}$. However, at intermediate temperatures, they were governed by the details of vibrations of the atoms and for a long time could only be determined from experiments. For a given temperature, $C_{\mathrm{v}}$ and $C_{\mathrm{p}}$ decrease with increasing pressure. From figures 2 and 3 , one can see that the influence of temperature on heat capacity is much more significant than that of pressure.

In figure 4, we present the effect of temperature and pressure on the volume expansion coefficient, $\alpha$ for PbSe and PbTe compounds. It is shown that at a given pressure, $\alpha$ increases sharply with the increase of temperature up to about $100 \mathrm{~K}$ and gradually approaches a linear increase at higher temperatures. It can be seen that

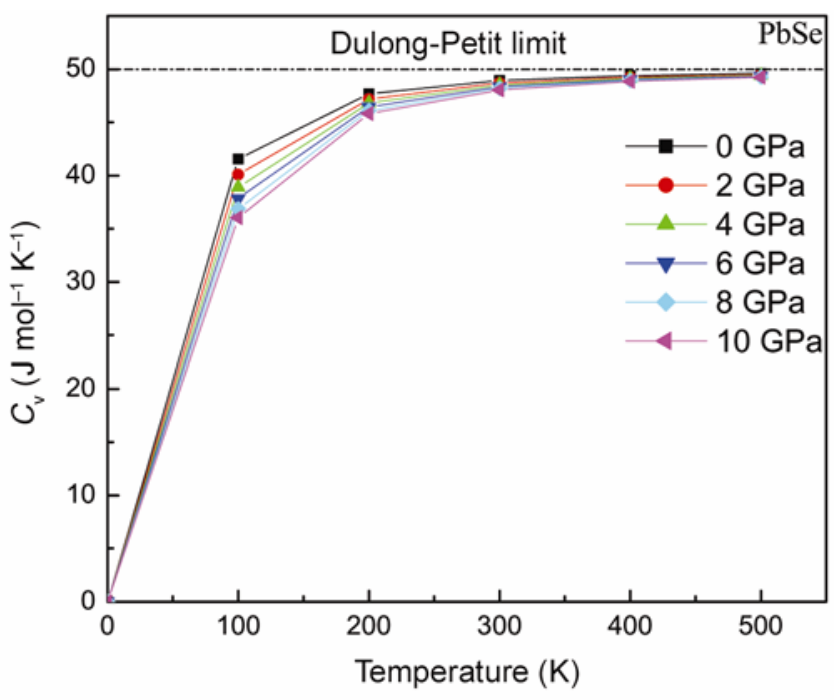

Figure 2. Variation of heat capacity, $C_{\mathrm{v}}$ of PbSe as a function of temperature at several pressures.

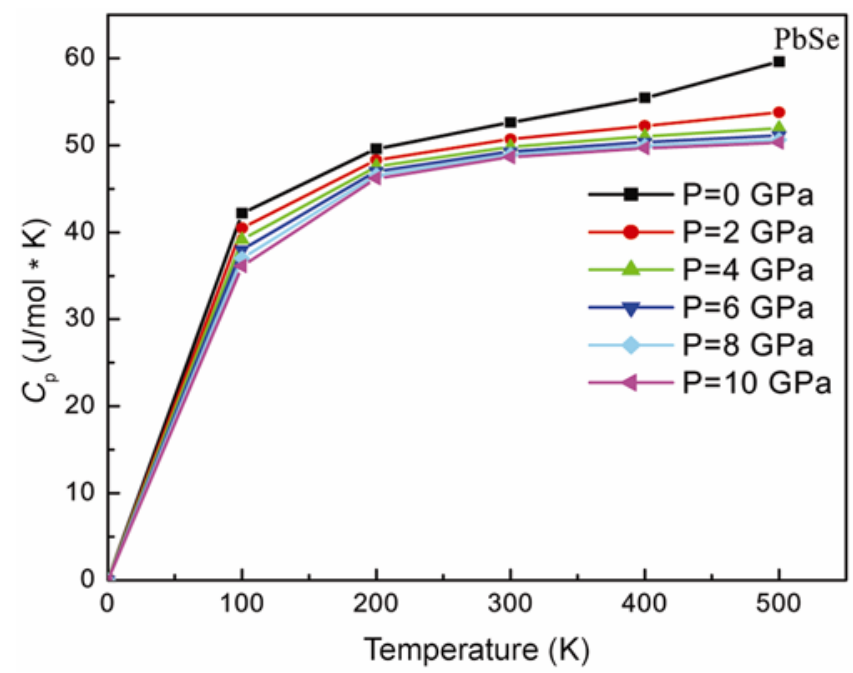

Figure 3. Variation of heat capacity, $C_{\mathrm{p}}$ of PbSe as a function of temperature at several pressures. thermal expansion coefficient, $\alpha$ decreases dramatically with the increase in pressure. As the pressure increases, the increase of $\alpha$ with temperature becomes smaller, especially at high temperatures.

The calculated properties at different temperatures are very sensitive to the vibrational effects. In the quasiharmonic Debye model, the Debye temperature is a key parameter. Finally, the pressure dependence of the Debye temperature is calculated and plotted in figure 5 . It is found that the Debye temperature, $\theta_{\mathrm{D}}$ increases with applied pressure, especially, at low pressure region. Compared with figure 1 , one can see that the compressibility increase leads to Debye temperature decrease. This result is in accordance with the fact that Debye temperature is proportional to the bulk modulus and that a hard material exhibits a high Debye temperature. It is also
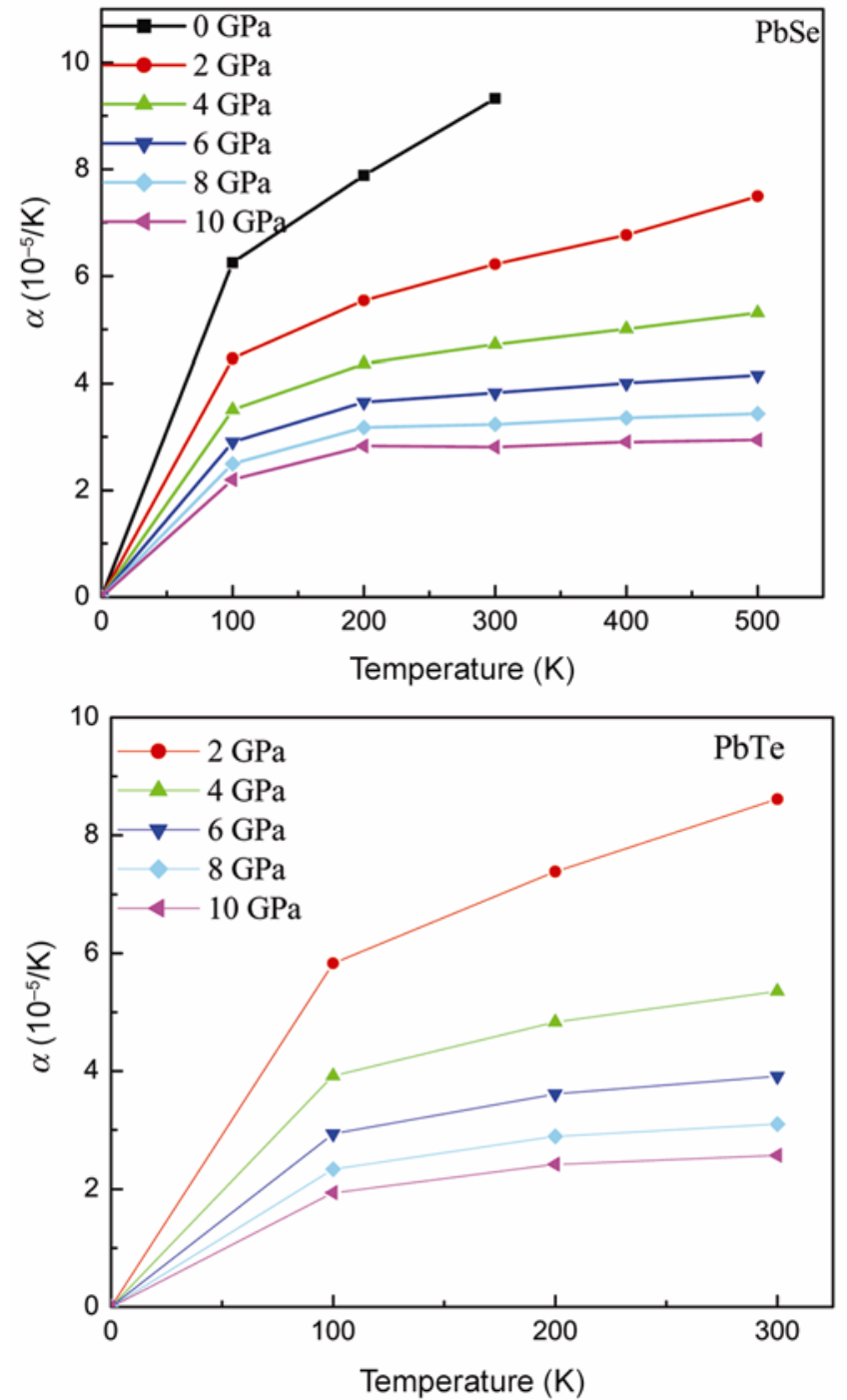

Figure 4. Variation of volume expansion coefficient, $\alpha$ of $\mathrm{PbSe}$ and $\mathrm{PbTe}$ as a function of temperature at several pressures. 

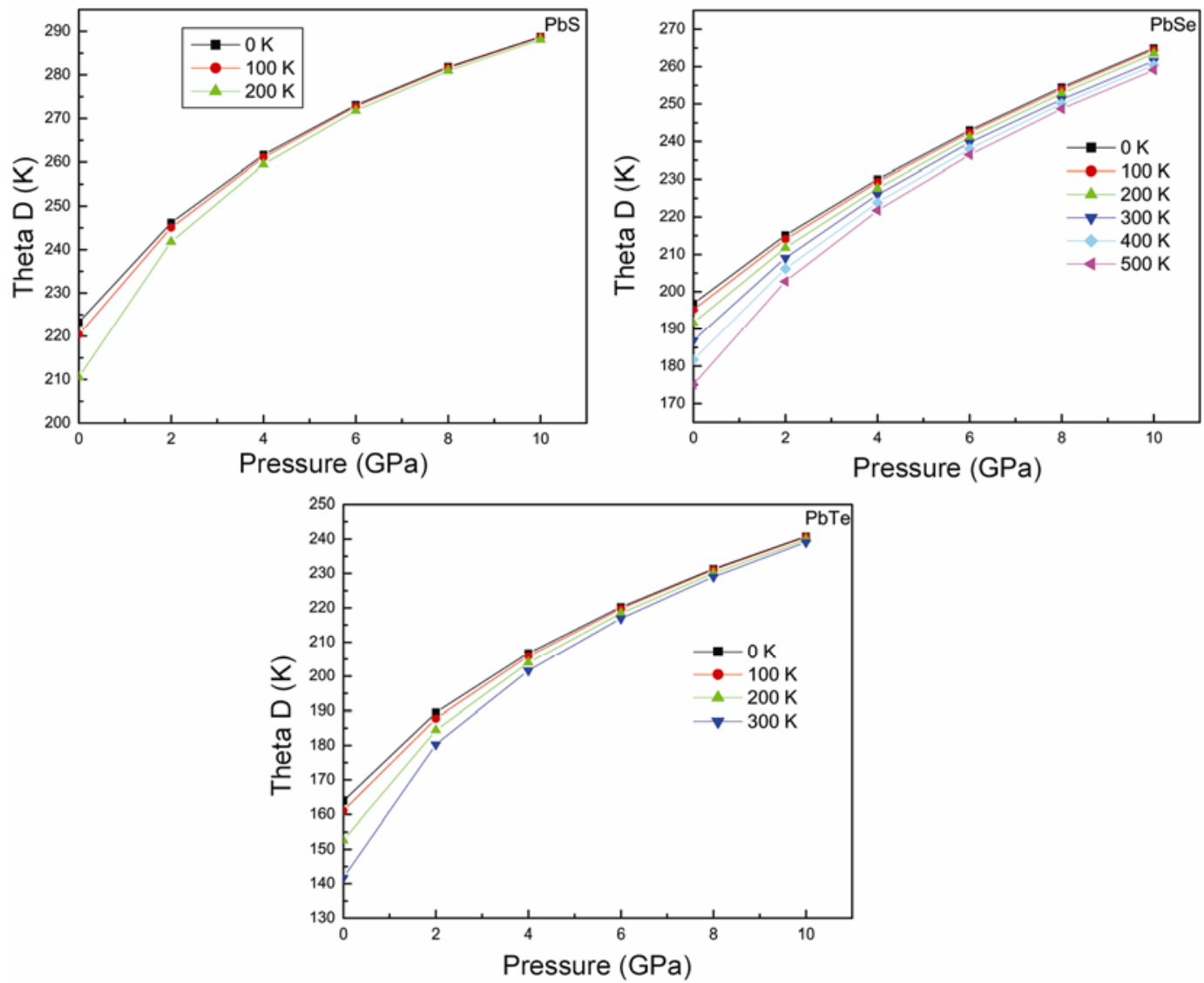

Figure 5. Variation of Debye temperature of $\mathrm{PbS}, \mathrm{PbSe}$ and $\mathrm{PbTe}$ as a function of pressure at several temperatures.

shown that when the pressure is constant, the Debye temperature decreases with temperature.

\section{Conclusions}

In summary, the present work reports a theoretical study of structural, electronic and thermal properties for lead chalcogenides PbS, PbSe and PbTe by using the FP-LAPW method within DFT. The main results can be summarized as follows:

(I) The calculated lattice constants and bulk modulus of the compounds are in good agreement with the experimental data and theoretical results.

(II) Our results regarding the band gap of the binary compounds are found to be in reasonable agreement with the theoretical data.

(III) Through the quasi-harmonic Debye model, the dependence of the lattice parameter, bulk modulus, heat capacity, volume expansion coefficient and Debye temperature, on temperature and pressure have been obtained successfully.

The present study, especially, thermal properties would be helpful for future experimental and theoretical investigations.

\section{References}

Ahuja R et al 1996 Phys. Rev. B54 10419

Albanesi et al 2000 Phys. Rev. B61 16589

Anderson O K 1975 Phys. Rev. B42 3060

Andreev A A 1968 J. Phys. C4 50

Besson J M et al 1968 Phys. Rev. 173699

Blaha P et al 2008 WIEN2K: An augmented plane wave plus local orbitals program for calculating crystal properties (Vienna, Austria: Karlheinz Schwarz, Techn. Universitat)

Blanco M A et al 2004 Comput. Phys. Commun. 15857

Blanco M A et al 1996 J. Molec. Struct. Theochem. 368245

Cohen M L and Chelikowsky J R 1989 Electronic structure and optical properties of semiconductors. Springer Series in Solids States Sciences (Berlin: Springer-Verlag) 2nd edn, Vol. 75

Cowley R A 1965 Philos. Mag. 11673

Dalven R (ed.) 1973 Solid state physics (New York: Academic) Vol. 28, p. 179

Debye P 1912 Ann. Phys. 3989

Delin A et al 1998 Int. J. Quant. Chem. 69349

Flórez M et al 2002 Phys. Rev. B66 144112

Francisco E et al 2001 Phys. Rev. B63 094107

Gupta V P and Ravindra N M 1980 Phys. Status Solidi. (b) 10715

Hohenberg P and Kohn W 1964 Phys. Rev. 136 B864

Hummer K et al 2007 Phys. Rev. B75 195211

Kohn W and Sham L S 1965 Phys. Rev. 140 A1133 
Lach-Hab M et al 2002 J. Phys. Chem. Solids 63833

Madelung $\mathrm{O}$ et al (eds) 1983 Numerical data and functional relationships in science and technology. Landolt-Bornstei, New Series (Berlin: Springer) Vol. 17

Martinez G 1973 Phys. Rev. B8 4686

Murnaghan F D 1944 Proc. Natl. Acad. Sci. USA 305390

Murase K 1980 J. Phys. Soc. Jpn. 49 (Suppl.) 725

Perdew J P and Wang Y 1986 Phys. Rev. B33 8800

Perdew J P et al 1996 Phys. Rev. Lett. 773865
Poirier J P 2000 Introduction to the physics of the Earth's interior (Oxford: Cambridge University Press) p. 39

Preier H 1979 Appl. Phys. 20189

Ravindra P et al 1997 Phys. Rev. B56 6851

Tauber R N et al 1966 J. Appl. Phys. 374855

Wei S and Zunger A 1997 Phys. Rev. B55 13605

Zaoui A et al 2009 Mater. Chem. Phys. 114650

Zhang Y et al 2009 Phys. Rev. B80 024304

Zogg H et al 1994 Opt. Eng. 331440 